\title{
EL DISEÑO DE LA "CoRe" PARA INICIAR LA IDENTIFICACIÓN, EXPLICITACIÓNY DESARROLLO DEL CPC DE UN PROFESOR DE BÁSICA PRIMARIA SOBRE EL NÚCLEO CONCEPTUAL DE LA NUTRICIÓN HUMANA
}

\section{"CoRe" Design to Start the Identification, Explicitation and Development of the PCK of Primary School Teachers on the Conceptual Core of Human Nutrition}

\section{O projeto da "CoRe" para iniciar a identificação explicação e o desenvolvimento do CPC de um professor de básica primária sobre o núcleo conceitual da nutrição humana}

\section{Boris Fernando Candela Rodríguez ${ }^{1}$ Diana Patricia Ruíz Taramuel ${ }^{2}$}

Fecha de recepción: 30 de septiembre de 2016

Fecha de aceptación: 1 de junio de 2017

\section{Resumen}

El presente trabajo de investigación documenta el proceso por el cual una profesora en ejercicio de grado quinto de Básica Primaria identifica, explicita y desarrolla el Conocimiento Pedagógico del Contenido (CPC) hipotético del núcleo conceptual de la nutrición humana. Para ello, se proponen actividades a realizar en dos fases: fundamentación e intervención. Así, la primera brinda la oportunidad a la profesora de conceptualizar e internalizar los siguientes elementos: el constructo del CPC, la herramienta de la $\mathrm{CoRe}^{3}$; dificultades/limitaciones y concepciones alternativas del tópico de la nutrición humana; aspectos de la pedagogía general; conocimiento del contexto; currículo estatal de la biología, y comprensión profunda del núcleo conceptual de la nutrición humana. La segunda fase se focaliza en asistir a la profesora en ejercicio durante la construcción consciente y progresiva del contenido que fundamenta a la CoRe de la nutrición humana, y posterior desarrollo del material de enseñanza de dicho núcleo conceptual en formato digital (blog). La metodología de investigación utilizada en este trabajo es de perspectiva cualitativa e interpretativa por

1 Profesor de la Universidad del Valle (IEP); miembro del grupo de investigación interinstitucional Ciencias, Acciones y Creencias UPN-UV (categoría A1). Correo electrónico: bofeca65@yahoo.com

2 Estudiante de Maestría en educación, énfasis en enseñanza de las ciencias naturales, Universidad del Valle. Correo electrónico: patric0267@ hotmail.es

3 Mulhall, Berry y Loughran (2003) conceptualizan la CoRe (Content Representation) como una herramienta metodológica que le brinda la posibilidad al profesor de tomar decisiones curriculares e instruccionales de manera reflexiva. Así, dicha herramienta durante la planeación de una lección ayuda a mediar la articulación consciente de los elementos del acto educativo: estudiantes, contenido y práctica de enseñanza. 
estudio de casos, en la cual la recolección de datos se lleva a cabo a lo largo de las anteriores fases, utilizando para esta tarea las siguientes fuentes documentales: observación participante de la serie de tareas de aprendizaje que han sido diseñadas, secuenciadas y temporalizadas por el investigador durante la fase de fundamentación; entrevista semiestructurada a la profesora; versiones de la CoRe, y diálogo informal.

Palabras clave: Conocimiento Pedagógico del Contenido; nutrición humana; docencia primaria; CoRe

\section{Abstract}

This research documents the process through which a fifth-grade primary school teacher identifies, makes explicit and develops the hypothetical Pedagogical Content Knowledge (PCK) of the conceptual core of human nutrition. For this purpose, we propose a number of activities to be performed in two phases: foundation and intervention. Thus, the first one gives the teacher the opportunity to conceptualize and internalize the following elements: PCK construction, the CoRe tool [3]; difficulties/limitations and alternative conceptions of the topic of human nutrition; aspects of general pedagogy; knowledge of the context; state biology curriculum, and deep understanding of the conceptual core of human nutrition. The second phase focuses on assisting the practicing teacher during the conscious and progressive construction of the content that is the basis of the CoRe for human nutrition, and the subsequent development of teaching material of said conceptual core in digital format (blog). The research methodology used in this work is of qualitative and interpretative perspective by case study, in which data collection is carried out throughout the previous phases, using the following documentary sources for this task: participant observation of the series of learning tasks that have been designed, sequenced and temporalized by the researcher during the foundation phase; semi-structured interview with the teacher; CoRe versions, and informal dialogue.

Keywords: Pedagogical Content Knowledge (PCK); human nutrition; primary teaching; core

\section{Resumo}

O presente trabalho de pesquisa apresenta o processo pelo qual uma professora em exercício de quinto ano de básica primária identifica, explicita e desenvolve o Conhecimento Pedagógico do Conteúdo (CPC) hipotético do núcleo conceitual da nutrição humana. Para isso, propõem-se atividades a realizar em duas fases: fundamentação é intervenção. Assim, a primeira dá oportunidade à professora de conceitualizar internalizar os seguintes elementos: o construto do CPC, a ferramenta da CoRe, dificuldades/limitações e concepções alternativas do tópico da nutrição humana; aspectos da pedagogia geral; conhecimento do contexto; currículo estadual da biologia e compreensão profunda núcleo conceitual da nutrição humana. A segunda fase está focalizada em assistir à professora durante a construção consciente e progressiva do conteúdo que fundamenta a CoRe da nutrição humana, e posterior desenvolvimento do material de ensino desse núcleo conceitual em formatos digital (blog). A metodologia de pesquisa utilizada neste trabalho é a perspectiva qualitativa e interpretativa por estudos de casos, na que a coleta de dados foi realizada durante as anteriores fases, utilizando para essa tarefa as seguintes fontes documentais: observação participante da série de tarefas de aprendizagem planejadas, sequenciadas e temporalizadas pelo pesquisador durante a fase de fundamentação; entrevista semiestruturada à professora; versões da CoRe e diálogo informal.

Palavras-chave: conhecimento pedagógico do conteúdo; nutrição humana; docência primária; CoRe 


\section{Introducción}

En Colombia, a partir de la formulación de la Ley 115 de 1994, en su capítulo tres y articulo $118^{4}$, se puede evidenciar de manera manifiesta que en la escuela primaria existen dos tipos de profesores en ejercicio, a saber: aquellos cuya área de acreditación es en educación (licenciados con formación en diferentes disciplinas) y los que pertenecen a otros campos del conocimiento (por ejemplo, ingenieros, abogados, sociólogos, entre otros). Desde luego, como lo afirma Appleton (2002), los profesores de la escuela primaria deben asumir las tareas de planeación, enseñanza y reflexión post-lección de las diferentes áreas de este nivel de escolaridad a partir de su sistema de conocimiento, creencias y valores que han construido a lo largo de etapas como aprendiz, profesor en formación y en ejercicio.

Conviene subrayar que los estudios focalizados en el aprendizaje y la enseñanza de las ciencias en la escuela primaria (por ejemplo, biología, química, física) dejan ver que aquellos profesores que se desempeñan en estos grados les falta confianza para planeary enseñar las ciencias (Appleton, 2003). De hecho, esta situación probablemente se deba a que las bases del conocimiento ${ }^{5}$ para la enseñanza ejemplar de un tópico específico con las que ellos enfrentan su práctica educativa no están en coherencia con el cuerpo de conocimientos que subyacen al movimiento de las actuales reformas en la educación en ciencias.

Así mismo, las investigaciones acerca de la enseñanza de las ciencias en educación primaria han mostrado que los conocimientos que poseen los docentes de primaria sobre el Conocimiento Pedagógico del Contenido (CPC) de las ciencias es limitado (Appleton, 2003; Appleton, 2008;

4 La Ley 115 de febrero 8 de 1994, Ley General de Educación, expresa lo siguiente: "Ejercicio de la docencia por otros profesionales. Por necesidades del servicio, quienes posean título expedido por las instituciones de educación superior, distinto al de profesional en educación o licenciado, podrán ejercer la docencia en la educación por niveles y grados, en el área de su especialidad o en un área a fin. Estos profesionales podrán también ser inscritos en el Escalafón Nacional Docente, siempre y cuando acrediten estudios pedagógicos en el país o en el extranjero, en una facultad de educación o en otra unidad académica responsable de la formación de educadores, con una duración no menor de un año" (cap. 3, art. 118).

5 Shulman (1987) conceptualizó como bases del conocimiento para la enseñanza las siguientes categorías: conocimiento de la disciplina; conocimiento de la pedagogía general; conocimiento pedagógico del contenido; conocimiento del currículum; conocimiento de los educandos y sus características; conocimiento de los objetivos, las finalidades, los valores educacionales y de sus fundamentos filosóficos e históricos.
Nilsson y Loughran, 2012). Adicionalmente, se considera que ellos no tienen confianza para diseñar e implementar ambientes de aprendizaje de tópicos del currículum de las ciencias. De hecho, quizá una de las causas de esta situación sea la falta del dominio de las disciplinas de las ciencias y su articulación con la pedagogía. De ahí que muchos maestros de la escuela primaria para salir adelante en la enseñanza de las ciencias toman prestadas estrategias y modelos de enseñanza provenientes de otras áreas de conocimiento.

Ahora bien, en las últimas décadas la comunidad del campo de la educación en ciencias aumentó el interés en indagar sobre cómo ayudar a los profesores de la escuela primaria a desarrollar el Conocimiento Pedagógico del Contenido (Nilsson y Loughran, 2012). Sin embargo, ellos son conscientes que el desarrollo del cPc es un proceso complejo determinado por el contenido para ser enseñando, el contexto en el cual el contenido es enseñado y la forma en la que los profesores reflexionan sobre la experiencia de enseñanza. De ahí que resulte clave la afirmación que sustenta si los profesores en formación están listos para ser exitosos en crear ambientes de aprendizaje en los cuales la disciplina y la pedagogía son integradas en formas que promuevan el aprendizaje, por lo cual ellos deberían de experimentar esos mismos ambientes durante sus cursos de formación (Magnusson, Krajcik y Borko, 1999).

El anterior presupuesto nos lleva a considerar que muchos de los profesores que se desempeñan en la escuela primaria evitan enseñar los tópicos de las ciencias. Sin embargo, en el caso en que lo hagan, ellos enfrentan dicho proceso utilizando rutinas, técnicas, estrategias y modelos de enseñanza provenientes de otras disciplinas del plan de estudios (Appleton y Kindt, 1997).

En este sentido, los educadores de profesores de ciencias han llegado al consenso que a pesar de los débiles antecedentes disciplinares y pedagógicos específicos con los que los profesores de ciencias de primaria enfrentan el ciclo instruccional (planeación, enseñanza y reflexión), muchos de ellos diseñan actividades de aprendizaje con la intención de salir adelante en el proceso de socialización y transformación de sus estudiantes. Pese a estos esfuerzos profesionales, la comprensión conceptual e integrada de los tópicos del currículum de las ciencias no viene siendo alcanzada por los estudiantes de la escuela primaria, como lo esperan los movimientos de las actuales reformas curriculares (Nichols y Koballa, 2006).

A partir de las anteriores asunciones los investigadores de educación en ciencias han llegado a un consenso, el cual hace referencia al hecho de que los profesores en

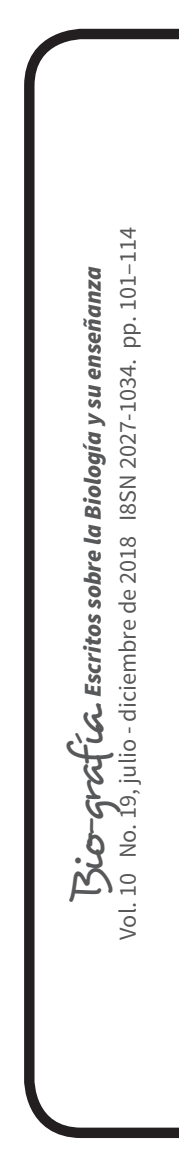


ejercicio de la escuela primaria necesitan alcanzar un desarrollo profesional que esté en coherencia con los marcos teóricos que subyacen a las actuales reformas de los programas de educación y movimientos curriculares que se están llevando a cabo. En otras palabras, se reconoce la necesidad que ellos continúen identificando y desarrollando su Conocimiento Pedagógico del Contenido de las ciencias (CPC). Definitivamente este desarrollo les brindaría la capacidad para diseñar, implementar y evaluar ambientes de aprendizaje cuyo fin central será el alcance y la comprensión conceptual e integrada de los tópicos del currículum de las ciencias por parte de los estudiantes, para que puedan tomar decisiones informadas en los diferentes escenarios en los que participen (Appleton, 2003).

En coherencia con lo anterior y con el propósito deliberado que un profesor de grado quinto de primaria comience a identificar y desarrollar el cPc de la biología, se ha tomado la decisión de abordar en este estudio el diseño de la enseñanza del núcleo conceptual, la nutrición humana, como punto de inicio de dicha tarea de desarrollo profesional. Esta determinación obedece a que este núcleo se vincula con otros tópicos estructurantes dentro y a través de los diferentes grados, situación que le permitiría al profesor diseñar un currículum coherente en biología, que quizá ejercería una fuerte influencia en el aumento de la comprensión conceptual de sus estudiantes.

En este sentido, para que el profesor de la escuela primaria (sujeto de investigación) comience a identificar y desarrollar su CPC de la biología, se considera necesario que desarrolle de manera reflexiva y deliberada el instrumento de la Representación del Contenido (CoRe) acerca de la nutrición humana. Desde luego, Hume y Berry (2011, 2013) han evidenciado que la construcción reflexiva del instrumento CoRe por parte de los profesores ejerce una fuerte influencia sobre su desarrollo profesional, dado que dicha tarea les permite identificar y desarrollar las bases de conocimiento para la enseñanza de las ciencias.

Finalmente esta actividad de desarrollo profesional le brinda la oportunidad al profesor de la escuela primaria hacer explícitas y transformar sus concepciones alternativas sobre la disciplina, la enseñanza y el aprendizaje de la nutrición humana. Desde luego, el conjunto de actividades de formación tiene como propósito deliberado que el cpc de la biología comience a evolucionar de forma progresiva hacia los presupuestos formulados por los actuales programas de formación del profesor de este nivel de escolaridad (Abell, Appleton y Hanusin, 2010).

Tomando como punto de referencia los anteriores presupuestos, en este estudio se aborda la siguiente pregunta de investigación: ¿De qué manera la construcción reflexiva y deliberada del instrumento CoRe le permite a un profesor de la escuela primaria identificar, explicitar y desarrollar el CPC hipotético de la nutrición humana?

\section{Metodología}

Con el propósito de documentar el pensamiento y acciones inteligentes, llevadas a cabo por la profesora, y al diseñar el currículum de la enseñanza de la nutrición humana, se decidió orientar esta investigación desde un enfoque de tipo cualitativo por estudio de caso de naturaleza instrumental (Stake, 1999). Dicho enfoque permite representar la forma como la profesora de la escuela primaria comienza a identificar, explicitar y desarrollar el CPC del núcleo de la nutrición humana.

Ahora bien, el caso bajo consideración (profesora de la escuela primaria) se selecciona direccionado por los siguientes criterios: 1) ganancia de aprendizaje; 2) disponibilidad de tiempo para el desarrollo de tareas de campo; 3) acceso y permanencia en el campo durante el tiempo necesario; 4) disposición y aptitud por parte del sujeto de investigación; 5) tiempo de experiencia como docente de Básica Primaria superior a cuatro años, y 6) desempeñarse actualmente como profesor de grado quinto o haberse desempeñado como docente de este grado al menos en dos ocasiones.

Una vez establecido el conjunto de criterios para la selección del caso necesario y para definir su indagación, se presentó la propuesta a los profesores de los grados cuarto y quinto, con fin de motivarlos a participar en el estudio (3 mujeres y 1 hombre). Luego de la socialización de la propuesta solamente la profesora ${ }^{6}$ de grado quinto manifestó interés por participar en el proyecto.

6 La profesora realizó sus estudios profesionales en una institución universitaria de Cali (Universidad del Valle), en donde obtuvo su título como licenciada en Biología y Química hace diez años. Cuenta con once años de experiencia docente, ocho de ellos vinculada al sector oficial como docente de Básica Primaria. A la fecha ha realizado diferentes cursos de formación, diplomados y capacitaciones relacionados con su área de formación inicial; además, ha estado orientada por los formadores del programa del Ministerio de Educación Nacional Todos a Aprender (PTA). 


\section{Obtención de datos ${ }^{7}$}

Los datos de esta investigación fueron obtenidos a través de varias actividades de formación, las cuales, para efectos de organización y descripción, se agruparon en dos momentos denominados "fase de fundamentación" y "fase de intervención". Así, la primera fase se focalizó en que la profesora de la escuela primaria en ejercicio conceptualiza e internaliza los siguientes elementos: el constructo del CPC, la herramienta de la CoRe; dificultades/limitaciones y concepciones alternativas del tópico de la nutrición humana; aspectos de la pedagogía general; conocimiento del contexto; currículo estatal de la biología, y comprensión profunda del núcleo conceptual de la nutrición humana. Esta actividad de formación tuvo como fin central que la profesora comenzara a internalizar de manera sinérgica los anteriores elementos a lo largo de la práctica del diseño del ambiente de aprendizaje del tópico de la nutrición humana. En cuanto a la segunda fase, esta se focalizó en asistir a la profesora en ejercicio durante la construcción consciente y progresiva del contenido que fundamenta la CoRe de la nutrición humana, y posteriormente se desarrolló el material de enseñanza de dicho núcleo conceptual en formato digital (blog).

Conviene subrayar que los datos de este estudio fueron recogidos a través de fuentes documentales, tales como: observación participante durante la construcción de la CoRe, entrevistas semiestructurada, relatos narrativos, diálogos informales y diarios reflexivos. Esta estrategia permitió realizar una triangulación por fuente (Denzin, 1989), la cual da confiabilidad y validez a la serie resultados de naturaleza cualitativa.

\section{Análisis de datos}

Con la intención de construir una teoría natural que explique de qué manera la construcción reflexiva del instrumento metodológico de la CoRe le permite a una profesora de la escuela primaria identificar, explicitar y desarrollar el CPC de la nutrición humana, se decidió

7 Es importante señalar que aunque la recolección de datos y el análisis se presentan como tareas separadas, estas se encuentran estrechamente relacionadas. De hecho autores como Eisenhardt (1989) se refieren a ellas en términos de solapamiento. Dicha situación se explica debido al ejercicio que se lleva a cabo durante la recolección de datos, es decir, simultáneamente a esta tarea el investigador examina y analiza, al realizar anotaciones de campo sobre las interpretaciones e impresiones que se van generando a lo largo de la actividad, lo cual le permitiría explorar otros aspectos.

8 La primera fase se desarrolló durante los meses de Octubre y Noviembre del año 2015. En cuanto la segunda fase se llevó a cabo a lo largo de los meses de febrero y mayo de 2016. analizar los datos desde la perspectiva de la teoría fundamentada de Strauss y Corbin (2002). A partir de estos referentes, en conjunción con el marco conceptual del cPC de las ciencias y desde el enfoque de Magnusson, Krajciky Borko (1999), se llevó a cabo los procesos de codificación abierta, axial y selectiva; para ello se realizan las siguientes tareas analíticas:

- Lectura sistemática de las diferentes versiones de la CoRe diseñada por la profesora en ejercicio con el propósito de recoger evidencia que apoyara la identificación, explicitación y desarrollo de los componentes del CPC en el tópico de la nutrición humana. Por tanto, se revisó detalladamente el contenido de cada uno de los ítems de las diferentes versiones de las CoRe diseñadas y se compararon con las unidades de análisis con sentido independiente, que provenían de otras fuentes documentales, tales como: observación participante durante la construcción de la CoRe, entrevista semiestructurada, relatos narrativos, diálogos informales y diarios reflexivos.

- Codificación de las unidades de análisis que estructuran las anteriores fuentes documentales.

- Lectura y relectura del conjunto de códigos asociados a las diferentes unidades de análisis con el propósito de evidenciar patrones de ocurrencia.

- Fragmentación de las unidades de análisis con el fin de reunir los códigos cuyo patrón de ocurrencia presentan similitudes en sus propiedades; de esta manera, se pudo generar las dos categorías de este estudio, el cPc y la CoRe (véase tabla 1). Conviene destacar que las propiedades de las unidades de análisis de mayor ocurrencia están alineadas con las respectivas características de los elementos que configuran el cPc desde la perspectiva de Magnusson, Krajcik y Borko (1999).

- El examen y comparación de las unidades básicas de análisis permitió la identificación de similitudes y diferencias en las propiedades, al admitir su adscripción a las categorías del cPc y la CoRe.

- Verificación de la pertenencia de las unidades de análisis tanto en las categorías de la CoRe como en las del CPC a las que fueron adscriptos, mediante su revisión.

- Una nueva revisión de la colección de unidades de análisis que había sido adscripta a las respectivas subcategorías de las categorías del cPc y la CoRe, con el fin de dar confiabilidad a dicha tarea.

- El cPC para este estudio es considerado como la categoría central o medular, la cual articula de manera semántica las subcategorías que configuran la CoRe. Es decir, esta tiene la capacidad de vincular proposicionalmente las unidades de análisis 
adscriptas a sus subcategorías con las de la CoRe. Como consecuencia de esta situación se produce una serie de generalizaciones naturalísticas o elementos teóricos, los cuales son desarrollados para generar un marco teórico que brinde la posibilidad de describir y comprender la identificación, explicitación y desarrollo del CPC del tópico nutrición humana en la profesora de la escuela primaria (véase tabla 1).
- Una nueva revisión a la colección de unidades de análisis que han sido adscriptas, tanto a las subcategorías del CPC como a las subcategorías de la CoRe, con el propósito deliberado de desarrollar teóricamente cada uno de los cinco elementos que configuran el CPC del tópico de la nutrición humana que la profesora de la escuela primaria ha comenzado a identificar, explicitary desarrollar a lo largo de este estudio.

Tabla 1. Relación selectiva entre las categorías del CPC y la ReCo

\begin{tabular}{|c|c|c|}
\hline \multirow{10}{*}{ 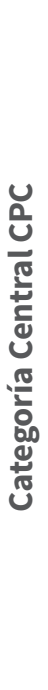 } & Subcategorías & Categoría CoRe \\
\hline & \multirow[t]{2}{*}{ Orientaciones hacia la enseñanza de la ciencia } & 2. ¿Por qué es importante que los alumnos sepan esta idea? \\
\hline & & $\begin{array}{l}\text { 7. ¿Cuáles procedimientos de enseñanza emplea? (y las razones } \\
\text { particulares de su uso con esta idea) }\end{array}$ \\
\hline & \multirow[t]{2}{*}{ Conocimiento y creencias acerca del currículo de la ciencia } & 1. ¿Qué intenta que aprendan los alumnos alrededor de esta idea? \\
\hline & & $\begin{array}{l}\text { 3. ¿Qué más sabe respecto a esta idea (y qué no incluye en sus } \\
\text { explicaciones a sus alumnos)? }\end{array}$ \\
\hline & $\begin{array}{l}\text { Conocimiento y creencias acerca de las estrategias } \\
\text { instruccionales para la enseñanza de la ciencia. }\end{array}$ & $\begin{array}{l}\text { 7. ¿Cuáles procedimientos de enseñanza emplea? (y las razones } \\
\text { particulares de su uso con esta idea) }\end{array}$ \\
\hline & \multirow[t]{3}{*}{$\begin{array}{l}\text { Conocimiento y creencias acerca de la comprensión de los } \\
\text { estudiantes de un tópico específico de la ciencia }\end{array}$} & $\begin{array}{l}\text { 4. ¿Cuáles son las dificultades/limitaciones relacionadas con la } \\
\text { enseñanza de esta idea? }\end{array}$ \\
\hline & & $\begin{array}{l}\text { 5. ¿Qué conocimientos acerca del pensamiento de los alumnos } \\
\text { influyen en su enseñanza de esta idea? }\end{array}$ \\
\hline & & 6. ¿Qué otros factores influyen en su enseñanza de esta idea? \\
\hline & $\begin{array}{l}\text { Conocimiento y creencias acerca de la evaluación en la } \\
\text { alfabetización científica }\end{array}$ & $\begin{array}{l}\text { 8. ¿Qué formas específicas de evaluación del entendimiento o de } \\
\text { la confusión de los alumnos emplea alrededor de esta idea? }\end{array}$ \\
\hline
\end{tabular}

Fuente: Candela (2016).

\section{Resultados}

El proceso de codificación abierta, axial y selectiva que se aplicó a la extensa información contenida en el conjunto de fuentes documentales permitió establecer una serie de generalizaciones naturalísticas. Dichas ideas están alineadas con las subcategorías de la categoría del CPC circunscrita a la perspectiva de Magnusson, Krajcik y Borko (1999) (véase la tabla 1); además se vincularon semánticamente con las subcategorías que configuran la CoRe. Estos vínculos fueron representados a través de proposiciones y macroestructuras textuales, que son el mecanismo por el cual se materializó la teoría generada desde el proceso de ordenamiento conceptual.

Así, la teoría que describe y explica el proceso a través del cual la profesora en ejercicio de biología comenzó a identificar, explicitar y desarrollar los elementos del CPC que encarnan las decisiones curriculares e instruccionales de la enseñanza de la biología es la siguiente:

\section{Orientación hacia la enseñanza de la ciencia}

Este componente del cpc describe la percepción que tienen los profesores con respecto a los propósitos y metas de enseñanza de un tópico específico. Es importante esclarecer que dichas orientaciones no están definidas en sí mismas por las estrategias que se utilizan, sino por los propósitos con las que estas se emplean. En este caso, inicialmente se llega a considerar que, dada la formación académica de la profesora y los marcos teóricos y metodológicos que subyacen a su formación universitaria, ella podría tener una orientación hacia la enseñanza de corte constructivista. Sin embargo, a través de los diálogos, la revisión de materiales se constata que las metas de enseñanza estaban centradas en la transmisión de hechos científicos y en su replicación por parte de los estudiantes. Fue así como se encontró que las principales actividades y estrategias desarrolladas consistían en ejercicios de transcripción y lectura. Poe esto, esta característica es relacionada con la fuerte influencia que tienen las áreas de Lengua Castellana y Matemáticas para determinar el 
trabajo escolar durante este ciclo de enseñanza. Según lo expresado por la profesora, existe una preocupación generalizada en el colectivo de profesores de este nivel por desarrollar durante los tiempos establecidos la totalidad de los contenidos y logros del currículo de estas. Sin embargo, aunque ellos focalizan su trabajo en dichas áreas, los resultados obtenidos en las pruebas estandarizadas (Saber) no corresponden con los esfuerzos que se realizan permanentemente. En consecuencia, esta situación genera aún más tensión y preocupación, convirtiéndose así en un hecho que lleva a los profesores a intensificar su trabajo escolar en estas áreas. La anterior asunción se evidencia en el siguiente testimonio:

En algunas ocasiones nosotros nos atrasamos en los temas de las materias y tomamos la decisión de desarrollar los temas de las áreas fundamentales (español y matemáticas) (...) cambiamos internamente los horarios (...) en ocasiones solo trabajamos español y matemáticas en toda una jornada. Así, podemos cumplir con el programa... esta presión la tenemos sobre todo los profesores de grados tercero y quinto, ya que nuestros estudiantes deben presentar las Pruebas Saber, nos preocupan los resultados, por eso les reforzamos mucho. Sin embargo, en otros grados también pasa lo mismo, es decir se trabajan más esas dos materias. (Entrevista a Lucia, 05 de febrero de 2016).

En relación con los procedimientos de instrucción para la enseñanza de las ciencias naturales, se evidenció que el criterio usado por la profesora es exclusivamente de carácter convencional fundamentado por la experiencia. Desde luego, se estableció que el único insumo al que recurre para determinar dichos procedimientos es el plan de área elaborado por los profesores de secundaria expertos. Naturalmente, debido a que los profesores de primaria no participan durante del plan en cuestión, ellos desconocen los fundamentos teóricos y metodológicos que soportan la toma de decisiones curriculares e instruccionales.

Ahora bien, a lo largo del proceso de acompañamiento de construcción y elaboración de la CoRe se alcanzó un logro importante relacionado con el reconocimiento del rol del estudiante en el aula de clase. De hecho, esta perspectiva no era un elemento desconocido para la profesora; así que la labor del programa de educación fue la de retomar los conocimientos de su formación pedagógica y enfocarlos nuevamente hacia su práctica educativa. En este caso se observa en la CoRe final y en la secuencia de enseñanza elaborada por la profesora que la variable conocimiento previo de los estudiantes resultó ser significativa para la organización y secuenciación de una serie de actividades de aprendizaje dirigidas a la articulación de este conocimiento con la nueva información adquirida.

Es preciso destacar que durante este ejercicio y gracias a la revisión permanente de los materiales diseñados por la profesora ella pudo percatarse que no todos los temas que configuran cada una de las grandes ideas pueden ser abordados desde un enfoque centrado en el estudiante. Con esto, este trabajo llevó a la docente a relacionar de manera consciente los diferentes elementos de la enseñanza en función de la naturaleza de la idea y del propósito de enseñanza. La siguiente cita ilustra la anterior situación:

si tienes en cuenta todos los elementos de enseñanza y piensas en la mejor manera que éstos empiecen a jugar o a articularse para favorecer el aprendizaje de tus estudiantes; es evidente que tu forma de ver la enseñanza cambia. Si eliges una perspectiva de enseñanza constructivista, no te alejas de los contenidos ni tampoco ellos dejan de ser importantes para la construcción y desarrollo de ideas. En algunos casos, podrás plantear situaciones de aprendizaje centradas en el estudiante y en otras es posible que tu como profesor debas asumir el papel central para explicar conceptos abstractos. (Entrevista a Lucia, 05 de febrero de 2016).

\section{Conocimiento y creencias acerca del currículum de la ciencia}

Esta subcategoría comprende las metas de aprendizaje conceptuales y las grandes metas de la formación en ciencias naturales ${ }^{9}$. Adicionalmente se evidenciaron las decisiones curriculares que toma el profesor a través de algunas acciones, tales como la selección, secuenciación, temporalización de los temas y el diseño de materiales curriculares alineados con los objetivos y necesidades de los estudiantes.

Teniendo en cuenta la respuesta dada por la profesora al ítem que recoge esta categoría, se pudo evidenciar que ella presentó varios obstáculos y restricciones para poder identificar, conceptualizar y hacer explícito el conjunto de ideas que configuran el núcleo conceptual de la nutrición humana. De hecho, la profesora en lugar de dar solución

9 Según lo establecido por el Ministerio de Educación Nacional en el documento n.‥3, "Estándares Básicos de competencia”(2006), las grandes metas de la formación en ciencias en la Educación Básica y Media son: a) el desarrollo del pensamiento científico; b) el desarrollo de la capacidad de seguir aprendiendo ; c) el desarrollo de la capacidad de valorar críticamente la ciencia, y d) aportar a la formación de hombres y mujeres miembros activos de una sociedad. 
a este ítem decidió en un primer momento esquivarlo, al abordar otros interrogantes del instrumento.

Ahora bien, en el momento en que ella decidió dar respuesta al primer interrogante de la CoRe planteó una secuenciación y conceptualización de ideas que no se hallan en coherencia con el conocimiento sustantivo y sintáctico que se encuentra representado en los estándares básicos de competencia. Probablemente esta restricción se da como consecuencia a la falta de una comprensión profunda del conocimiento sustantivo y sintáctico de este núcleo conceptual ${ }^{10}$. Este aspecto se convierte en un obstáculo para el diseño de un ambiente de aprendizaje que medie de manera eficiente en la comprensión de la nutrición humana para los estudiantes de grado quinto de primaria. La siguiente unidad de análisis tomada del diario reflexivo de la profesora amplía lo citado:

escoger una secuencia de ideas relacionadas con la nutrición humana me pareció una tarea bastante difícil, especialmente porque uno se da cuenta de que no tiene claridad sobre el tema y tampoco cuenta con los conocimientos para poder descomponer el tema en unidades más pequeñas [...] Al enfrentarme a esta tarea sentí muchas dudas [...] sentía que mi conocimiento y habilidades eran cuestionados, pensaba que lo que había trabajado por tantos años no era lo adecuado. (Diario reflexivo de Lucía, 11 de febrero de 2016).

Tomando en consideración el segundo interrogante (¿Qué más sabe respecto a esta idea y qué no incluye en sus explicaciones a sus alumnos?), se vio claramente que, si bien existe en el currículo estatal una relación estrecha entre el conocimiento del contenido (sustantivo y sintáctico) y la coherencia curricular horizontal y vertical, esta era desconocida por la profesora. Así, en relación a la coherencia vertical se evidenció que para ella no existe diferencias significativas entre el contenido disciplinar y la forma como este debe ser representado a lo largo de los grados escolares. En otras palabras, representa e implementa los mismos contenidos y actividades de aprendizaje para los diferentes grados de la escolaridad. Desde luego, durante la primera etapa del diseño se pudo observar la falta de competencia para delimitar los contenidos dentro de la zona de desarrollo proximal de los estudiantes, de acuerdo con algunos criterios, tales como naturaleza de la gran idea y lenguaje multinivel de las ciencias.

10 La profesora realizó la primera selección y secuenciación de las principales ideas que estructuran la enseñanza de la nutrición humana, apoyándose solo en la sabiduría que le otorga su experiencia como docente y como estudiante.
Con respecto a la articulación horizontal, se encontró que dicha coherencia curricular tampoco se refleja en la selección y secuenciación de las grandes ideas. Esto obedece no solo a la carencia de elementos de referencia derivados de la coherencia vertical, sino al desconocimiento de la estructura curricular -ejes articuladores ${ }^{11}-\mathrm{y}$ los procesos de enseñanza que subyacen a la disciplina. Por tanto, la falta de dominio sobre este referente estructural traerá como consecuencia obstáculos para que los estudiantes puedan establecer relaciones conceptuales, así como vínculos con tópicos de otras disciplinas en un mismo nivel de escolaridad.

Finalmente, durante la elaboración de las diferentes versiones de la CoRe se pudo observar cómo aquellas dificultades eran superadas de forma progresiva ${ }^{12}$. Esta tarea no fue sencilla, particularmente porque la profesora percibía que su experiencia e incluso sus conocimientos disciplinares eran frecuentemente cuestionados. Claro está que esta situación en lugar de representar un obstáculo se convirtió en un factor motivante para el desarrollo de la tarea misma. Es así como ella, al identificar sus propias dificultades, se interesó mucho más por aclarar y explicitar sus ideas, integrando a sus construcciones elementos teóricos revisados y discutidos previamente. La articulación de estos aspectos puede observarse en la CoRe final, en la cual se presenta un registro de ideas más estructuradas y amplias, pues buscan articular el núcleo conceptual de la nutrición humana de forma vertical y horizontal al currículo (con respecto a esto, véase el link http://profesoraciencias5.blogspot.com.co/p/herramienta.html). La siguiente idea representa el anterior presupuesto:

uno se acostumbra a definir conceptos y temas... y cree que está bien...pero si uno se pone a pensar realmente en cómo estos temas te van a ayudar a construir un concepto mayor, entonces allí es cuando hallas sentido a lo que trabajas, no los ves aislados... en este caso las grandes ideas que uno selecciona para construir la CoRe. (Diario reflexivo de Lucia, 11 de febrero de 2016).

11 El documento de Estándares Básicos de Competencia, del Ministerio de Educación Nacional (2008), establece que los ejes articuladores para las ciencias naturales son: la aproximación al conocimiento natural, el manejo de conocimientos propios de las ciencias y el desarrollo de compromisos personales y sociales. Cada uno de estos ejes se compone de un grupo de acciones que permitan el alcance de los estándares por conjuntos de grados.

12 Conviene subrayar que a lo largo de la fase de intervención la profesora orientada por el investigador de este estudio diseñó tres versiones de la CoRe para la enseñanza de la gran idea, nutrición humana. 


\section{Conocimiento y creencias acerca de las estrategias instruccionales para la enseñanza de la ciencia}

Para precisar algunos de los hallazgos de esta categoría, se establece los siguientes dominios: estrategias instruccionales generales y estrategias instruccionales específicas. En relación con la primera, y de acuerdo con descripciones previas, es claro que la profesora orientó sus estrategias desde un modelo de enseñanza transmisionista ${ }^{13}$, situación que muy difícilmente les permitirá a los estudiantes modificar sus ideas alternativas y construir de manera progresiva e integrada las grandes ideas que estructuran el núcleo conceptual de estudio. Probablemente esta orientación de enseñanza convencional se encuentra relacionada con el poco dominio que tiene la profesora sobre el contenido de la biología. Además, este esquema de enseñanza le permite tener control de los roles y dinámicas del aula sin llegar a sentirse amenazada. Dicha situación se ejemplifica en la viñeta.

\begin{abstract}
[L]os niños saben que en clase de naturales siempre copiamos y ellos están preparados para hacerlo. Yo copio en el tablero, les doy tiempo para que transcriban, pero si veo que están haciendo desorden pues borro [...] por eso siempre tienen que estar haciendo algo [...] coloco otra actividad sopa de letras, hacer un dibujo, un taller. Luego les copio la tarea o la dicto [...] Si se me olvida, ellos preguntan [...] ya están acostumbrados. (Entrevista a Lucia, 13 mayo de 2016).
\end{abstract}

Conviene subrayar que durante el desarrollo de la primera CoRe la profesora se limitó a enumerar una serie de acciones y procedimientos generales restrictivos con los cuales pretendió desarrollar los temas de cada gran idea. Así mismo, en un ejercicio posterior, formuló una serie de cuestionamientos orientados hacia la adquisición exclusiva de conocimiento factual. Desde luego, ninguna de estas acciones favorece el aprendizaje por comprensión conceptual.

En relación con el segundo dominio, se encontró que este se constituye por los siguientes aspectos: el conocimiento de las representaciones específicas, la capacidad y habilidad que tienen los profesores para poder crear dichas representaciones, y el conocimiento del uso de cada una de estas en los diferentes momentos de aprendizaje.

13 La profesora, asume otro tipo de orientación para la enseñanza de los temas que configuran las áreas de Matemáticas y Lenguaje; centrando sus actividades en un modelo de corte constructivista sociocultural.
Vale la pena destacar que, en el comienzo del programa de formación, si bien la profesora en ejercicio utilizó un conjunto de representaciones con el fin de estructurar las actividades de aprendizaje, estas no se encuentran articuladas a un propósito de aprendizaje específico. Es decir que su uso potencial como herramienta para ayudar al estudiante a conocer y vincular sus concepciones alternativas con los marcos teóricos nuevos presentados durante la actividad de aula es de un nivel bajo. En ese sentido, este tipo de representaciones y formulaciones del contenido no le brindan la oportunidad al estudiante de aproximar su lenguaje social intuitivo al lenguaje social y científico.

Dadas las anteriores circunstancias y con el propósito de superar las dificultades encontradas en torno a la falta de conocimiento y dominio de las estrategias instruccionales para la enseñanza, se enfrentó a la profesora en ejercicio ante las siguientes situaciones: a) lectura individual de documentos de trabajo asignados por los investigadores; b) discusión y puesta en común de los diferentes elementos teóricos encontrado, y c) indagaciones realizadas por la profesora de forma independiente y por iniciativa propia. A partir de estos elementos de referencia, la profesora estableció una estructura organizativa centrada en un enfoque constructivista y, metodológicamente, recurrió al modelo del Ciclo de Aprendizaje (constituido por las fases de exploración, introducción de vocablos y aplicación de conceptos). Esta decisión trajo consigo, desde luego, una serie de repercusiones significativas asociadas de manera directa a la selección, diseño, secuenciación y temporalización de las actividades de aprendizaje. Evidencia del desarrollo de este aspecto del cPc de la nutrición humana es el cuerpo de conocimiento que sustenta el material instruccional diseñado por la profesora junto con el contenido de la "CoRe", el cual está disponible a través del siguiente link: www.profesoraciencias5.blogspot.com.co/.

\section{Conocimiento y creencias acerca de la comprensión de los estudiantes de la nutrición humana}

Esta categoría describe el conocimiento que deben tener los profesores sobre los requisitos necesarios para que los estudiantes aprendan un tema específico. En este sentido, es fundamental que el profesor identifique y haga conscientes las dificultades o limitaciones conceptuales, cognitivas, contextuales y demás aspectos implicados durante el proceso de instrucción.

Ahora bien, dados los antecedentes del caso en estudio, se suponía que la profesora tendría varios inconvenientes para dar solución al interrogante que recoge esta 
categoría. De hecho, esta hipótesis se confirmó al observar la falta de desarrollo de este este ítem en las primeras versiones de la CoRe. Sin embargo, estos resultados no fueron un impedimento para la ejecución de la tarea; por el contrario, se convirtió en un factor promotor para el aprendizaje. Fue así como el interés de la profesora le condujo a realizar de manera independiente la búsqueda, revisión y lectura de diversos artículos relacionados con el tema. Naturalmente estos recursos, sumados a las reflexiones producto de los continuos diálogos con el investigador; le permitieron a ella identificar las dificultades y limitaciones de los estudiantes en torno al tópico de estudio. Al establecer una clasificación de las mismas, se focalizó, se diseñó y se ordenaron las actividades de aprendizaje que tienden hacia la superación de las dificultades en cuestión. El siguiente testimonio permite evidenciar el anterior presupuesto:

Cuando revisaba los materiales de lectura, me di cuenta que podía agrupar en algunas categorías las dificultades asociadas con la enseñanza de la nutrición [...] si bien, a cada grupo le pertenecen unas subcategorías ligadas, por ejemplo, al grado de compresión de los procesos o de relación, si hablamos de sistemas; creo que el hecho de tener un panorama general o de grandes categorías me ayudará a diseñar las actividades específicas necesarias para abordarlas. El ejercicio de clasificación me llevo a definir cuatro grupos: a) variedad de esquemas conceptuales para explicar la nutrición humana (sistemas que participan en el proceso y falta de relación entre ellos); b) representaciones alternativas de los sistemas (conocimiento anatómico limitado); c) ideas alternativas (para explicar los diferentes procesos y funciones de los órganos y sistemas); d) poco o ningún uso de actividades que incluyan los diferentes niveles de organización de los seres vivos (por ejemplo, los estudiantes no relacionan la función energética de los nutrientes con el metabolismo celular...). (Entrevista a Lucia, 13 de mayo de 2016).

Otro aspecto significativo de esta categoría se relaciona con la naturaleza abstracta de los contenidos que configuran el tópico de la nutrición humana y su relación con la formación y permanencia de ideas alternativas. En este caso señalamos como un avance importante en la comprensión de esta naturaleza abstracta el hecho que la profesora enunció y, en algunos casos, describió las dificultades asociadas a los diferentes niveles de organización y representación de las ciencias naturales. Estos referentes le aportaron al diseño del conjunto de situaciones de aprendizaje orientadas hacia la exploración, descripción y relación de los diferentes niveles. Sin embargo, no se descartó que factores asociados a las formas de razonamiento y de pensamiento de los estudiantes puedan actuar como obstáculos en la comprensión de esta naturaleza abstracta. Por tanto, es fundamental que estos se hagan explícitos y conscientes para orientar a los profesores en la toma de decisiones instruccionales.

\section{Conocimiento y creencias acerca de la evaluación de la gran idea de la nutrición humana}

Inicialmente la posición de la profesora en torno a los objetivos y usos de la evaluación no se hizo explícita; sin embargo, se identificó a través de su discurso una serie de características que permiten establecer que estos probablemente se encuentran alineados con un enfoque pedagógico de carácter sumativo. De esta manera su intención era verificar, al final del proceso de enseñanza-aprendizaje, el grado de precisión que tienen los estudiantes en relación a una serie de contenidos conceptuales desarrollados. Así, si los resultados cumplieron con las expectativas de la profesora - reproducción y memorización de contenidos científicos-, el estudiante obtuvo una nota de aprobación, en caso contrario este sería desaprobado.

En concordancia con estos propósitos, se observó que los instrumentos de evaluación utilizados por la profesora no requirieron un alto nivel de exigencia cognitiva, pues se centran en la retención y memorización de la información. Adicionalmente, ella señaló que además de verificar rápidamente los aprendizajes e informar oportunamente a sus estudiantes su calificación, el uso de estos instrumentos le permitieron contar con tiempo extra para llevar a cabo actividades de aprendizaje pendientes en otras asignaturas. Podemos evidenciar este aspecto en la siguiente declaración:

\begin{abstract}
al final de cada unidad realizó una evaluación, es muy complicado hacer una serie de evaluaciones durante el desarrollo de ésta. Tienes que calificar y sacar nota de otras nueve asignaturas... sería una locura sacar cualquier otra cantidad de notas... tomas la opción más rápida para evaluar, diseñando una prueba de selección múltiple con única respuesta, preguntas de falso o verdadero o de completar...no te desgastas elaborando un tipo de prueba más compleja. Además, los estudiantes terminan rápidamente... cuentas con tiempo para calificar y registrar en las planillas sin dejar acumular más trabajo [...]. (Entrevista a Lucia, 27 de abril de 2016).
\end{abstract}

Otra característica que conviene señalar está relacionada con la influencia de las prácticas evaluativas llevadas a cabo por la profesora en ejercicio dentro del aula. Por una parte, estas son empleadas como formas de regular 
el comportamiento y garantizar el orden durante el desarrollo de las clases. Por otro lado, tener el control quizá le ayuda a la profesora a legitimar su estatus dentro del aula, garantizando que los estudiantes no la lleven por fuera de la zona de confort.

Por otro lado, la discusión colegiada entre la profesora y el investigador, en conjunto con la lectura analítica de la serie de artículos focalizados en la evaluación formativa, le permitió a la primera explicitar e internalizar las ideas en torno a los usos y propósitos de esta clase de evaluación formativa. En este sentido, la profesora identificó una serie de aspectos que la orientaron a realizar un cambio de perspectiva, desde el señalamiento del error hacia un pensamiento dirigido a la formación integral de los estudiantes, a través de la comprensión de cómo ellos aprenden y progresan a lo largo de su escolaridad.

Con base en estas premisas, uno de los primeros elementos destacados por la profesora es el carácter continuo de la evaluación, el cual le permitiría identificar los logros y dificultades asociados al desarrollo de cada una de las grandes ideas. A partir de ello, podría tomar decisiones para intervenir y mejorar el proceso de la enseñanza-aprendizaje antes de que este termine. Este hecho la llevó a considerar la necesidad de incluir una variedad de actividades de aprendizaje y diversos procedimientos de evaluación (formales e informales) que den cuenta del proceso por medio de un monitoreo permanentemente.

Un aspecto que generó a la profesora cierto grado de tensión e incomodidad se relaciona con la puesta en escena de esta práctica de evaluación en el aula de clase. Ella expresó que el uso de la evaluación formativa resultaría ser una tarea bastante exigente, pues consiste en detectar, orientar y adaptar las estrategias para un grupo particular de estudiantes sin descuidar las necesidades de cada uno de ellos; además, articular todos estos elementos a unos propósitos de enseñanza no es una tarea sencilla. Sin embargo, este aspecto, que fue señalado inicialmente como un inconveniente, luego fue considerado por ella como una oportunidad de aprendizaje, la cual le permitió contrastar sus experiencias previas del uso de la evaluación con los elementos teóricos desarrollados a lo largo de la construcción colaborativa de la CoRe. De esta manera, la docente contaría con nuevas evidencias para sustentar los cambios instruccionales a los que ellos dieran lugar.

\section{Conclusiones}

El uso del instrumento metodológico de la CoRe, en conjunción con las actividades de cualificación desarrolladas, permitió analizar las concepciones alternativas relacionadas con la nutrición humana, la enseñanza y el aprendizaje de las ciencias, con las que llegó la profesora en ejercicio al programa de educación, las cuales inciden de manera significativa en la forma como ella representa y formula el conocimiento científico para un grupo particular de estudiantes. En ese sentido, se describe a continuación las principales conclusiones obtenidas en cada una de las categorías del CPC de la nutrición humana.

En relación con la categoría "Orientación hacia la enseñanza de las ciencias", se observó que el enfoque constructivista que la profesora adoptó le permite identificar, explicitar y evidenciar las relaciones de causa y efecto entre los diferentes elementos de la enseñanza y la naturaleza abstracta de los contenidos. Este ejercicio le brindó la posibilidad de determinar que, en algunas situaciones, de acuerdo con la naturaleza del contendido y las metas de enseñanza, se puede adoptar una orientación hacia la enseñanza diferente a la constructivista (por ejemplo, centrada en el profesor), con el propósito de enlazar el aprendizaje por comprensión conceptual de los contenidos de naturaleza abstracta.

Con respecto a la categoría "Conocimiento y creencias acerca del currículum de la biología", se destaca el desarrollo y apropiación de una serie de elementos conceptuales que le permitieron a la profesora seleccionary secuenciar las ideas que configuran el núcleo conceptual de la nutrición humana de forma coherentemente curricular dentro y a lo largo de los diferentes grados de escolaridad. Dicho desarrollo le brindó la posibilidad de diseñar un material instruccional que asistiera al estudiante en la comprensión conceptual de la gran idea de nutrición humana. Aunque se presentaron dificultades para que la profesora pudiera desarrollar las grandes ideas del núcleo de estudio, esta situación fue asumida por ella como una oportunidad para avanzar en su conocimiento profesional, lejos de ser un obstáculo.

El análisis de los referentes conceptuales y metodológicos de perspectiva constructivista sociocultural le ayudó a la profesora a reevaluar y modificar las dinámicas y roles establecidos en el aula de clases. A partir de allí se observó un cambio significativo en los elementos de la categoría conocimiento y creencias acerca de las estrategias instruccionales. Adicionalmente, estos referentes le proporcionaron una serie de evidencias para definir una estructura organizativa de actividades y tareas orientadas hacia el aprendizaje por comprensión conceptual e integrada de los contenidos escolares. También cabe señalar que la profesora utilizó a lo largo del desarrollo de la CoRe los diferentes niveles de organización de los seres vivos como un criterio para el diseño de las actividades de aprendizaje, cuyo propósito central descansa en asistir a los estudiantes en la construcción de una visión sistémica de la nutrición humana. 
A través de las discusiones permanentes en torno a los diferentes documentos de trabajo y reflexiones sobre la práctica educativa, se observó cómo la profesora empieza a evidenciar nuevos elementos de análisis para poder resolver los interrogantes y tareas de esta y otras categorías del cPc. Tal es el caso de la categoría conocimiento y creencias de la comprensión de los estudiantes de un tópico específico de las ciencias, cuyo interés inicial se dirige hacia la búsqueda e identificación de una variedad de ideas alternativas, dificultades conceptuales y cognitivas que tienen los estudiantes para comprender cada una de las grandes ideas. De esta manera, pudo centrarse en los aspectos fundamentales de cada una de estas para diseñar y secuenciar actividades específicas destinadas a su superación.

Finalmente, en la categoría "Creencias acerca de la evaluación en la alfabetización científica" se identificaron varios logros importantes con respecto a los usos y propósitos de la evaluación formativa. En este caso, la práctica pasó de ser una estrategia exclusiva de detección, señalamiento de errores y control a ser reconocida por su valor formativo. En este sentido, la profesora asumió la evaluación como una práctica de carácter continuo y dinámico, la cual permita monitorear y detectar oportunamente las dificultades y fortalezas del proceso de enseñanza-aprendizaje para establecer acciones de mejoramiento sobre la marcha.

De la misma manera, la profesora reconoció que el valor formativo de la evaluación aplica en doble sentido, es decir, esta le permite al profesor monitorear el nivel de comprensión y compromiso de sus estudiantes; igualmente, brinda la oportunidad para reflexionar sobre la práctica del diseño y la enseñanza de un contenido con el fin de tomar decisiones curriculares e instruccionales coherentes con las metas de las actuales reformas curriculares. Por tanto, es necesario incluir diversas actividades de aprendizaje y procedimientos de evaluación que den cuenta del nivel de desarrollo de los contenidos conceptuales, procedimentales y actitudinales de las ciencias.

Por último, se ha podido evidenciar que el diseño progresivo y colaborativo del instrumento metodológico de la CoRe le ofreció a la profesora en ejercicio la oportunidad de continuar identificando, explicitando y desarrollando los elementos que configuran el CPC de la nutrición humana. Así mismo, el desarrollo de dicho instrumento dentro del contexto problemático del diseño de un ambiente de aprendizaje específico a un contenido es probablemente una estrategia de formación potencialmente alta para comenzar a mejorar la enseñanza y aprendizaje de las ciencias en la escuela primaria.

\section{Referencias}

Abell, S. K., Appleton, K., y Hanuscin, D. L. (2010). Designing and Teaching the Elementary Science Methods Course. New York: Routledge.

Appleton, K. (2002). Science activities that Work: Perceptions of Primary School Teachers. Research in Science Education, 32, 390-410.

Appleton, K. (2003). How do Beginning Primary School Teachers Cope with Science? Toward and Understanding of Science Teaching Practice. Research in Science Education, 33, 1-25.

Appleton, K. (2008). Developing Science Pedagogical Content Knowledge Through Mentoring Elementary Teachers. Journal of Science Teacher Education, 19, 523-545.

Appleton, K., y Kindt, I. (1997). Research Monograph: Beginning Teachers' Practices in Primary Science in Rural Areas. Rockhampton, Queensland: Faculty of Education, Central Queensland University.

Candela, B. F. (2016). El diseño de la "ReCo": una estrategia para iniciar la identificación, explicitación y desarrollo del cpc de un tópico la química de profesores en formación inicial. Tecné, Episteme y Didaxis TED, 40, 23-25

Denzin, N. K. (1989). Strategies of Multiple Triangulation. The Research Act: A Theoretical Introduction to Sociological Methods. New York: McGraw Hill.

Eisenhardt, K. M. (1989). Building Theories from Case Study Research. Academy of Management Review, 4(4), 532-550.

Hume A., y Berry, A. (2013). Enhancing the Practicum Experience for Pre-service Chemistry Teachers Through Collaborative CoRe Design with Mentor Teachers. Research in Science Education, 43, 21072136.

Hume, A., y Berry, A. (2011). Constructing CoRes: A Strategy for Building Pск in Pre-service Science Teacher Education. Research in Science Education, 41, 341-355.

Magnusson, S., Krajcik, J., y Borko, H. (1999). Nature, Sources and Development of Pedagogical Content Knowledge. En J. Gess-Newsome y N. G. Lederman (Eds.), Examining pedagogical content knowledge (pp. 95-132). Dordrecht: Kluwer Academic Publishers. 
Mulhall, P., Berry, A., \& Loughran, J. (2003). Frameworks for representing science teachers' pedagogical content knowledge. In Asia-Pacific Forum on Science Learning and Teaching, 4, 1-25.

Nichols, S. E., y Koballa, T. (2006). Framing Issues of Elementary Science Teacher Education: Critical Conversations. En K. Appleton, (Ed.), Elementary science teacher education: International perspectives on contemporary issues and practice (pp. 1-12). Mahwah, NJ: Lawrence Erlbaum Associates.

Nilsson, P., y Loughran, J. (2012). Exploring the Development of Pre-service Science Elementary Teach- ers' Pedagogical Content Knowledge. Journal of Science Teacher Education, 23(7), 699-721.

Shulman, L. (1987). Knowledge and Teaching: Foundations of the New Reform Harvard. Educational Review, 57(1), 1-22.

Stake, R. (1999). Investigación con estudio de casos. Madrid: Morata.

Strauss, A., y Corbin, J. (2002). Bases de la investigación cualitativa: técnicas y procedimientos para desarrollar la teoría fundamentada. Medellín: Universidad de Antioquia. 


\section{Anexo 1. Tabla de la herramienta metodológica de la CoRe}

UNIVERSIDAD DEL VALLE

INSTITUTO DE EDUCACIÓN Y PEDAGOGÍA

MAESTRIA EN EDUCACIÓN (ÉNFASIS EN ENSEÑANZA DE LA CIENCIA)

BASE PARA LA ENTREVISTA (CoRe)

¿Cuáles son las ideas científicas que se encuentran en el centro del tópico “

"? Es decir, seleccione entre tres a cinco ideas en las que acostumbre a dividir la enseñanza del concepto . Se trata de que en ese conjunto de ideas estén reflejadas las más importantes del tema a impartir, o de sus precedentes.

Para cada una de estas ideas responda las siguientes preguntas:

\begin{tabular}{|c|c|c|c|}
\hline IDEAS/CONCEPTOS IMPORTANTES EN CIENCIAS & $\begin{array}{l}\text { Idea } \\
n .^{\circ} 1\end{array}$ & $\begin{array}{l}\text { Idea } \\
\text { n. }{ }^{\circ} 2\end{array}$ & $\begin{array}{l}\text { Idea } \\
\text { n. }{ }^{\circ} 3\end{array}$ \\
\hline 1. ¿Qué intenta que aprendan los alumnos alrededor de esta idea? & & & \\
\hline 2. ¿Por qué es importante que los alumnos sepan esta idea? & & & \\
\hline 3. ¿Qué más sabe respecto a esta idea (y qué no incluye en sus explicaciones a sus alumnos)? & & & \\
\hline 4. ¿Cuáles son las dificultades/limitaciones relacionadas con la enseñanza de esta idea? & & & \\
\hline $\begin{array}{l}\text { 5. ¿Qué conocimientos acerca del pensamiento de los alumnos influyen en su enseñanza de es } \\
\text { idea? }\end{array}$ & & & \\
\hline 6. ¿Qué otros factores influyen en su enseñanza de esta idea? & & & \\
\hline $\begin{array}{l}\text { 7. ¿Cuáles procedimientos de enseñanza emplea? (y las razones particulares de su uso } \\
\text { con esta idea). }\end{array}$ & & & \\
\hline $\begin{array}{l}\text { 8. ¿Qué formas específicas de evaluación del entendimiento o de la confusión de los alumnos } \\
\text { emplea alrededor de esta idea? }\end{array}$ & & & \\
\hline
\end{tabular}

